\title{
Geometric invariant theory and projective toric varieties
}

\author{
Nicholas Proudfoot ${ }^{1}$ \\ Department of Mathematics, University of Texas, Austin, TX 78712
}

Abstract. We define projective GIT quotients, and introduce toric varieties from this perspective. We illustrate the definitions by exploring the relationship between toric varieties and polyhedra.

\begin{abstract}
Geometric invariant theory (GIT) is a theory of quotients in the category of algebraic varieties. Let $X$ be a projective variety with ample line bundle $\mathrm{E}$, and $G$ an algebraic group acting on $X$, along with a lift of the action to $\mathrm{E}$. The GIT quotient of $X$ by $G$ is again a projective variety, along with a given choice of ample line bundle. With no extra work, we can consider varieties which are projective over affine, that is varieties can be written in the form Proj $R$ for a reasonable graded ring $R$. The purpose of this note is to give two equivalent definitions of projective GIT quotients, one algebraic in terms of the homogeneous coordinate ring $R$, and one more geometric, and to illustrate these definitions with toric varieties.
\end{abstract}

A toric variety may be defined abstractly to be a normal variety that admits a torus action with a dense orbit. One way to construct such a variety is to take a GIT quotient of affine space by a linear torus action, and it turns out that every toric variety which is projective over affine arises in this manner. Given the data of a torus action on $\mathbb{C}^{n}$ along with a lift to the trivial line bundle, we define a polyhedron, which will be bounded (a polytope) if and only if the corresponding toric variety is projective. We then use this polyhedron to give two combinatorial descriptions of the toric variety, one in the language of algebra and the other in the language of geometry.

Much has been written about toric varieties, from many different perspectives. The standard text on the subject by Fulton $[\mathrm{Fu}]$ focuses on the relationship between toric varieties and fans. The main difference between this approach and the one that we adopt here is that a fan corresponds to an abstract toric variety, while a polyhedron corresponds to a toric variety along with a choice of ample line bundle. In particular, there exist toric varieties

\footnotetext{
${ }^{1}$ Supported by the Clay Mathematics Institute Liftoff Program and the National Science Foundation Mathematical Sciences Postdoctoral Research Fellowship.
} 
which are not projective over affine, and which are therefore do not come from polyhedra. Since the primary purpose of this note is to introduce projective GIT quotients, we will avoid fans altogether. Two excellent treatments of toric varieties from the GIT perspective can be found in books by Dolgachev [Do, §12] and Miller and Sturmfels [MS, §10]. Each of these books takes a broader approach to GIT than we do in this note; Dolgachev has a more categorical perspective that goes beyond the projective case, and Miller and Sturmfels stress the fact that everything may be interpreted in the language of multigraded commutative algebra. What we lack here in depth and generality we hope to make up for with brevity and concreteness.

Acknowledgments. The author is grateful to Herb Clemens, Rob Lazarsfeld, and Ravi Vakil for organizing this conference, and for providing useful feedback during the preparation of this talk and paper.

\section{Geometric invariant theory}

Consider a graded noetherian algebra

$$
R=\bigoplus_{m=0}^{\infty} R_{m}
$$

which is finitely generated as an algebra over $\mathbb{C}$. The variety $X=\operatorname{Proj} R$ is projective over the affine variety $\operatorname{Spec} R_{0}$, and comes equipped with an ample line bundle $\mathrm{E}=\mathcal{O}_{X}(1)$. Furthermore, by [Ha, Ex. 5.14(a)], the integral closure (or normalization) of $R$ is isomorphic to the ring

$$
R^{\prime}=\bigoplus_{m=0}^{\infty} \Gamma\left(X, \mathrm{七}^{\otimes m}\right) .
$$

The most important example for our applications will be the following.

Example 1.1 Let $R=\mathbb{C}\left[x_{1}, \ldots, x_{n}, y_{0}, \ldots, y_{k}\right]$, with $\operatorname{deg} x_{i}=0$ and $\operatorname{deg} y_{j}=1$. Then Proj $R \cong \mathbb{C}^{n} \times \mathbb{C} P^{k}$, and $\mathrm{E}$ restricts to the antitautological bundle $\mathcal{O}(1)$ on $\{z\} \times \mathbb{C} P^{k}$ for all $z \in \mathbb{C}^{n}$.

Let $G$ be a reductive algebraic group. A good reference for general reductive groups is [FH], however the only groups that we will need for our applications in Section 2 are 
subgroups of the algebraic torus $\left(\mathbb{C}^{\times}\right)^{n}$. Suppose that we are given an action of $G$ on $R$ that preserves the grading; such an action induces an action of $G$ on $X=\operatorname{Proj} R$ along with a lift of this action to the line bundle $\mathrm{E}$. This lift is sometimes referred to as a linearization of the action of $G$ on $X$.

Definition 1.2 The variety $X / / G:=\operatorname{Proj}\left(R^{G}\right)$ is called the GIT quotient of $X$ by $G$, where $R^{G}$ is the subring of $R$ fixed by $G$.

The reader is warned that, while the action of $G$ on $\mathrm{E}$ is not incorporated into the notation $X / / G$, it is an essential part of the data. In particular, we will see in Example 1.5 that it is possible to change the linearization of an action and obtain a vastly different quotient.

Definition 1.2 is very easy to state, but not so easy to understand geometrically. Our next goal will be to give a description of $X / / G$ which depends more transparently on the structure of the $G$-orbits in $X$. Let $\mathrm{E}^{*}$ denote the line bundle dual to $\mathrm{E}$.

Definition 1.3 A closed point $x \in X$ is called semistable if, for all nonzero covectors $\ell \in \mathrm{E}_{x}^{*}$ over $x$, the closure of the $G$-orbit $G \cdot(x, \ell)$ in the total space of $\mathrm{E}^{*}$ is disjoint from the zero section. A point which is not semistable is called unstable. The locus of semistable points will be denoted $X^{s s}$.

Theorem 1.4 There is a surjective map $\pi: X^{s s} \rightarrow X / / G$, with $\pi(x)=\pi(y)$ if and only if the closures of the orbits $G \cdot x$ and $G \cdot y$ intersect in $X^{s s}$.

Proof: We will provide only a sketch of the proof of Theorem 1.4 for a more thorough argument, see [Do, Prop 8.1]. The projection from $R$ onto $R_{0}$ induces an inclusion of Spec $R_{0}$ into $\operatorname{Spec} R$, and the complement $\operatorname{Spec} R \backslash \operatorname{Spec} R_{0}$ fibers over $\operatorname{Proj} R$ with fiber $\mathbb{C}^{\times}$. The inclusion of $R^{G}$ into $R$ induces a surjection $\tilde{\pi}: \operatorname{Spec} R \rightarrow \operatorname{Spec} R^{G}$. Let $x$ be an element of $X$, and let $\tilde{x}$ be a lift of $x$ to $\operatorname{Spec} R \backslash \operatorname{Spec} R_{0}$. Then

$$
\begin{aligned}
x \in X^{s s} & \Longleftrightarrow \exists \text { a } G \text {-invariant section of } \mathrm{E}^{\otimes m} \text { not vanishing at } x \text { for some } m>0 \\
& \Longleftrightarrow \exists f \in R_{m}^{G} \text { not vanishing at } \tilde{x} \text { for some } m>0 \\
& \Longleftrightarrow \tilde{\pi}(\tilde{x}) \notin \operatorname{Spec} R_{0}^{G} \subseteq \operatorname{Spec} R^{G}
\end{aligned}
$$

hence $\tilde{\pi}(\tilde{x})$ descends to an element of Proj $R^{G}$. Since the inclusion of $R^{G}$ into $R$ respects the gradings on the two rings, this element does not depend on the choice of lift $\tilde{x}$, hence $\tilde{\pi}$ induces a surjection $\pi: X^{s s} \rightarrow \operatorname{Proj} R^{G}=X / / G$. Two points $x, y \in X^{s s}$ with lifts $\tilde{x}, \tilde{y} \in \operatorname{Spec} R$ 
lie in different fibers of $\pi$ if and only there exists a $G$-invariant function $f \in R_{>0}^{G}$ that vanishes at $\tilde{x}$ but not at $\tilde{y}$, which is the case if and only if the closures of the $G$ orbits through $x$ and $y$ in $X^{s s}$ are disjoint.

Our proof of Theorem 1.4 suggests that the variety Proj $R$ may itself be interpreted as a GIT quotient of Spec $R$ by the group $\mathbb{C}^{\times}$. Indeed, the grading on $R$ defines an action of $\mathbb{C}^{\times}$ on $R$ by the formula $\lambda \cdot f=\lambda^{m} f$ for all $f \in R_{m}$, and this induces an action of $\mathbb{C}^{\times}$on Spec $R$. Consider the lift of this action to the trivial line bundle $\operatorname{Spec} R \times \mathbb{C}$ given by letting $\mathbb{C}^{\times}$act on the second factor by scalar multiplication. The unstable locus for this linearized action is exactly the subvariety $\operatorname{Spec} R_{0} \subseteq \operatorname{Spec} R$, and $\operatorname{Proj} R$ is the quotient of $\operatorname{Spec} R \backslash \operatorname{Spec} R_{0}$ by $\mathbb{C}^{\times}$. This provides a geometric explanation of the irrelevance of the irrelevant ideal in the standard algebraic definition of Proj.

We conclude the section with an example that illustrates the dependence of a GIT quotient on the choice of linearization of the $G$ action on $X$.

Example 1.5 As in Example [1.1, let $R=\mathbb{C}\left[x_{1}, \ldots, x_{n}, t\right]$, with $\operatorname{deg} x_{i}=0$ for all $i$ and $\operatorname{deg} t=1$. Then $X \cong \mathbb{C}^{n}$, and $\mathrm{E}$ is trivial. Let $G=\mathbb{C}^{\times}$act on $R$ by the equations

$$
\lambda \cdot x_{i}=\lambda x_{i} \text { and } \lambda \cdot t=\lambda^{\alpha} t
$$

for some $\alpha \in \mathbb{Z}$. Geometrically, $G$ acts by scalar multiplication, and $\alpha$ defines the linearization. This action is not to be confused with the action of $\mathbb{C}^{\times}$on $R$ given by the grading.

Case 1: $\alpha \geq 1$. In this case, $R^{G}=\mathbb{C}$, and $X / / G=\operatorname{Proj} R^{G}$ is empty. For every element $(x, \ell) \in \mathrm{E}^{*}$, we have $\lim _{\lambda \rightarrow 0} \lambda \cdot(x, \ell)=(0,0)$, hence every $x \in X$ is unstable.

Case 2: $\alpha=0$. With the trivial linearization of the $G$ action on $X$, we have $R^{G}=\mathbb{C}[t]$, hence $X / / G=\operatorname{Proj} R^{G}$ is a point. The $G$ orbits in $\mathrm{E}^{*}$ are all horizontal, hence every point is semistable. Since every $G$ orbit in $X$ contains the origin of $\mathbb{C}^{n}$ in its closure, Theorem 1.4 confirms that the quotient is a single point.

Case 3: $\alpha=-1$. In this case, $R^{G}=\mathbb{C}\left[x_{1} t, \ldots, x_{n} t\right]$ is a polynomial ring generated in degree 1 , hence $X / / G=\operatorname{Proj} R^{G} \cong \mathbb{C} P^{n-1}$. We have $\lambda \cdot(x, \ell)=\left(\lambda x, \lambda^{-1} \ell\right)$, which limits to an element of the zero section of $\mathrm{E}^{*}$ if and only if $x=0$. Thus $X^{s s}=\mathbb{C}^{n} \backslash\{0\}$, and all $G$ orbits 
in $X^{s s}$ are closed, hence the GIT quotient is isomorphic to the quotient of $X^{s s}$ by $G$ in the ordinary topological sense.

Case 4: $\alpha<-1$. In this case we still get $X / / G \cong \mathbb{C} P^{n-1}$, but we now obtain $\mathbb{C} P^{n-1}$ in its $(-\alpha)$-uple Veronese embedding.

Note that in Example 1.5. Multiplying $\alpha$ by a positive integer $m$ corresponds to replacing the $G$-equivariant line bundle $€$ on $X=\mathbb{C}^{n}$ with its $m^{\text {th }}$ tensor power. In general, this operation will have the effect of replacing the resulting ample line bundle on the GIT quotient $X / / G$ by its $m^{\text {th }}$ tensor power, as well (as we saw in Case 4 ).

\section{Toric varieties}

In this section we introduce and analyze toric varieties, which we will think of as generalizations of Example 1.5 to higher dimensional tori. As in the previous section, we let

$$
X=\mathbb{C}^{n}=\operatorname{Proj} \mathbb{C}\left[x_{1}, \ldots, x_{n}, t\right]
$$

with $\operatorname{deg} x_{i}=0$ and $\operatorname{deg} t=1$. Fix an $n$-tuple $\alpha=\left(\alpha_{1}, \ldots, \alpha_{n}\right)$ of integers, and let $T^{n}=$ $\left(\mathbb{C}^{\times}\right)^{n}$ act on $R=\mathbb{C}\left[x_{1}, \ldots, x_{n}, t\right]$ by the equations

$$
\lambda \cdot x_{i}=\lambda_{i} x_{i} \text { and } \lambda \cdot t=\lambda_{1}^{\alpha_{1}} \ldots \lambda_{n}^{\alpha_{n}} t
$$

for $\lambda=\left(\lambda_{1}, \ldots, \lambda_{n}\right) \in T^{n}$. Thus we have the standard coordinate action of $T^{n}$ on $\mathbb{C}^{n}$, with a linearization to the trivial bundle given by $\alpha$.

Definition 2.1 A toric variety is a GIT quotient of $X$ by a subgroup $G \subseteq T^{n}$ for some $n$.

A toric variety $X / / G$ admits an action of the torus $T=T^{n} / G$ with a single dense orbit. A more standard approach to toric geometry is to define a toric variety to be a normal variety along with a torus that acts with a dense orbit, and then to prove that every such variety which is projective over affine arises from the construction of Definition 2.1. For the strictly projective case, see [Fu, $\S 3.4]$.

Consider the exact sequence

$$
1 \rightarrow G \rightarrow T^{n} \rightarrow T \rightarrow 1
$$


Differentiating at the identity, we obtain an exact sequence of complex Lie algebras

$$
0 \rightarrow \mathfrak{g} \rightarrow \mathfrak{t}^{n} \rightarrow \mathfrak{t} \rightarrow 0
$$

Let $\left\{e_{1}, \ldots, e_{n}\right\}$ be the coordinate vectors in $\mathfrak{t}^{n}$, and let $a_{i}$ be the image of $e_{i}$ in $\mathfrak{t}$. The vector space $\mathfrak{t}$ is equipped with an integer lattice $\mathfrak{t}_{\mathbb{Z}}=\operatorname{ker}(\exp : \mathfrak{t} \rightarrow T)$. Its dual $\mathfrak{t}^{*}$ therefore inherits a dual lattice, as well as a canonical real part $\mathfrak{t}_{\mathbb{R}}^{*}=\mathfrak{t}_{\mathbb{Z}}^{*} \otimes_{\mathbb{Z}} \mathbb{R}$. We now define the polyhedron

$$
\Delta=\left\{p \in \mathfrak{t}_{\mathbb{R}}^{*} \mid p \cdot a_{i} \geq \alpha_{i} \text { for all } i\right\},
$$

a subset of the real vector space $\mathfrak{t}_{\mathbb{R}}^{*}$.

There is a deep and extensive interaction between the toric variety $X / / G$ and the polyhedron $\Delta$. The $T$ orbits on $X$, for example, are classified by the faces of $\Delta$, with faces of real dimension $i$ corresponding to orbits of complex dimension $i$. If $\Delta$ is simple (exactly $\operatorname{dim}_{\mathbb{R}} \Delta$ facets meet at each vertex), then $X / / G$ is an orbifold [LT], and the Betti numbers of $X / / G$ are determined by the equation

$$
\sum_{i=0}^{d} b_{2 i}(X / / G) q^{i}=\sum_{i=0}^{d} f_{i}(\Delta)(q-1)^{i}
$$

where $d=\operatorname{dim}_{\mathbb{C}} X / / G=\operatorname{dim}_{\mathbb{R}} \Delta$, and $f_{i}(\Delta)$ is the number of faces of dimension $i$. This fact has been famously used by Stanley to characterize the possible face vectors of simple polytopes [St], and can be proven in many ways. One beautiful (though unnecessarily technical) proof uses the Weil conjectures; it amounts simply to observing that the right hand side of Equation (10) may be interpreted as the number of points on an $\mathbb{F}_{q}$ model of $X / / G$. For a more detailed discussion of Betti numbers, the Weil conjectures, and Stanley's theorem, see $[\mathrm{Fu}, \S 4.5$ and 5.6]. In this note, we will content ourselves with using $\Delta$ to describe the invariant ring $R^{G}$ and the semistable locus $X^{s s}$.

Let $C_{\Delta}$ be the cone over $\Delta$, that is

$$
C_{\Delta}:=\left\{(p, r) \in \mathfrak{t}_{\mathbb{R}}^{*} \times \mathbb{R} \mid r \geq 0 \text { and } p \in r \cdot \Delta\right\}^{c l},
$$

where $c l$ denotes closure inside of $\mathfrak{t}_{\mathbb{R}}^{*} \times \mathbb{R}$. The following figure illustrates the cone over an interval and the cone over a half line. Note that the closure is necessary to include the positive $x$-axis in the cone over the half line. Let $S_{\Delta}:=C_{\Delta} \cap\left(\mathfrak{t}_{\mathbb{Z}}^{*} \times \mathbb{Z}\right)$ be the semigroup consisting of all of the lattice points in $C$. We may then define the semigroup ring $\mathbb{C}\left[S_{\Delta}\right]$, an 

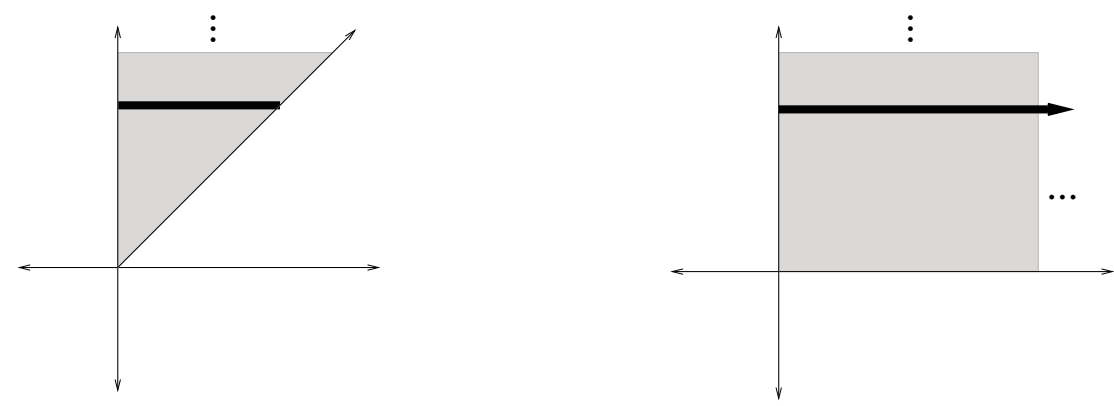

algebra over $\mathbb{C}$ with additive basis indexed by the elements of $S_{\Delta}$, and multiplication given by the semigroup law. This ring has a non-negative integer grading given on basis elements by the final coordinate of the corresponding lattice points. The following theorem provides a combinatorial interpretation of the homogeneous coordinate ring $R^{G}$ of $X / / G$.

Theorem $2.2 R^{G} \cong \mathbb{C}\left[S_{\Delta}\right]$.

Proof: Suppose that we are given an element $(p, r) \in S_{\Delta}$, and let $r_{i}=p \cdot a_{i}-r \alpha_{i} \in \mathbb{Z}_{\geq 0}$ for all $i$. To this element, there corresponds a $G$-invariant monomial $m_{(p, r)}=x_{1}^{r_{1}} \ldots x_{n}^{r_{n}} t^{r} \in R$. This correspondence defines a bijection from $S_{\Delta}$ to the monomials of $R^{G}$, which extends to a graded ring isomorphism $\mathbb{C}\left[S_{\Delta}\right] \cong R^{G}$.

For all $i \in\{1, \ldots, n\}$, let $F_{i}=\left\{p \in \Delta \mid p \cdot a_{i}=\alpha_{i}\right\}$. The set $F_{i}$ is the locus of points on $\Delta$ on which the $i^{\text {th }}$ defining linear form is minimized, and therefore it is a face of $\Delta$. If $\alpha$ is chosen generically, then $F_{i}$ will either be a facet or it will be empty. In general, however, $F_{i}$ may be a face of any dimension. The following theorem provides a combinatorial interpretation of the semistable locus $X^{s s}$.

Theorem 2.3 For any point $x \in X$, let $A=\left\{i \mid x_{i}=0\right\}$ be the set of coordinates at which $x$ vanishes. Then $x$ is semistable if and only if $\bigcap_{i \in A} F_{i} \neq \emptyset$.

Proof: In the proof of Theorem 1.4, we argued that $x$ is stable if and only if there is a positive degree element of $R^{G}$ that does not vanish at $x$. This will be the case if and only if there is $G$-invariant monomial of positive degree which is supported on the complement of $A$. By Theorem 2.2 $G$-invariant monomials of degree $r$ correspond to lattice points in $r \cdot \Delta$, and those that are supported on the complement of $A$ correspond to those that lie on $r \cdot F_{i}$ 
for all $i \in A$. Such a monomial exists if and only if $\bigcap_{i \in A} F_{i} \neq \emptyset$.

We conclude the section by using Theorems 2.2 and 2.3 to compute the toric varieties associated to an assortment of polytopes. An integer vector $a \in \mathfrak{t}_{\mathbb{Z}}^{*}$ is called primitive if it cannot be expressed as a multiple of another element $a^{\prime} \in \mathfrak{t}_{\mathbb{Z}}^{*}$ by an integer greater than 1 . In each of the following examples we will implicitly assume that the given polytope is cut out by the minimum possible number of linear forms $\left\{a_{1}, \ldots, a_{n}\right\}$ in the dual vector space, and that each of these forms is a primitive integer vector.

Example 2.4 Let $\Delta=\mathbb{R}^{+} \subset \mathbb{R}$ be the set of non-negative real numbers. Then $C_{\Delta}=\left(\mathbb{R}^{+}\right)^{2}$ and $\mathbb{C}\left[S_{\Delta}\right] \cong \mathbb{C}[x, t]$, with $\operatorname{deg} x=0$ and $\operatorname{deg} t=1$. This tells us that the associated toric variety is Proj $\mathbb{C}[x, t]=\mathbb{C}$. Geometrically, we have $T^{n}=T=\mathbb{C}^{\times}$, and $G$ is the trivial group, hence we are building $\mathbb{C}$ as a trivial GIT quotient of $\mathbb{C}$ itself. More generally, the toric variety associated to the positive orthant in $\mathbb{R}^{d}$ is $\mathbb{C}^{d}$, equipped with the trivial line bundle.

Example 2.5 Let $\Delta=[0,1]$ be the unit interval in $\mathbb{R}$. Then $S_{\Delta} \cong \mathbb{C}[x, y]$ is a polynomial ring in two variables of degree 1 , and the associated toric variety is $\mathbb{C} P^{1}$. Geometrically, $\mathbb{C}^{\times}$acts by scalars on $\mathbb{C}^{2}$, and the origin is the unique unstable point. More generally, the toric variety associated to the standard $d$-simplex in $\mathbb{R}^{d}$ is $\mathbb{C} P^{d}$ with its antitautological line bundle.

Example 2.6 Let $\Delta=[0, m] \subset \mathbb{R}$ for some positive integer $m$. The action of $\mathbb{C}^{\times}$on $\mathbb{C}^{2}$ and the semistable locus are unchanged from Example 2.5. hence the associated toric variety is again $\mathbb{C} P^{1}$. Its homogeneous coordinate ring $\mathbb{C}\left[S_{\Delta}\right]$, however, is isomorphic to the subring of $\mathbb{C}\left[S_{[0,1]}\right]$ spanned by homogeneous polynomials in degrees which are multiples of $m$. Hence the line bundle that we obtain on $\mathbb{C} P^{1}$ is the $m^{\text {th }}$ tensor power of the antitautological line bundle. More generally, dilating $\Delta$ by a positive integer $m$ corresponds to taking the $m^{\text {th }}$ tensor power of the ample line bundle on the toric variety. (See the end of Section 11)

Example 2.7 Let $\Delta=[0,1] \times[0,1] \subset \mathbb{R}^{2}$. This corresponds to an action of $\left(\mathbb{C}^{\times}\right)^{2}$ on $\mathbb{C}^{4}$, given in coordinates by

$$
(\lambda, \mu) \cdot\left(z_{1}, z_{2}, z_{3}, z_{4}\right)=\left(\lambda z_{1}, \lambda z_{2}, \mu z_{3}, \mu z_{4}\right)
$$

The unstable locus consists of the points where either $z_{1}=z_{2}=0$ or $z_{3}=z_{4}=0$, and the quotient of the semistable points by $\left(\mathbb{C}^{\times}\right)^{2}$ is isomorphic to $\mathbb{C} P^{1} \times \mathbb{C} P^{1}$. On the algebraic 
side, we have

$$
\mathbb{C}\left[S_{\Delta}\right] \cong \mathbb{C}[x, y, z, w] /\langle x z-y w\rangle,
$$

where $x, y, z$, and $w$ are generators in degree 1 corresponding to a cyclic ordering of the vertices of $\Delta$. In general, the toric variety corresponding to the product of two polytopes is isomorphic to the product of the corresponding toric varieties, in the Segré embedding.

\section{References}

[Do] I. Dolgachev. Lectures on Invariant Theory. London Mathematical Society Lecture Note Series 296, Cambridge University Press, 2003.

[Fu] W. Fulton. Introduction to toric varieties. Annals of Mathematics Studies, 131. Princeton University Press, 1993.

[FH] W. Fulton and J. Harris. Representation Theory: a First Course. Graduate Texts in Mathematics 129. Springer-Verlag, 1991.

[Ha] R. Hartshorne. Algebraic Geometry. Graduate Texts in Mathematics 52, Springer-Verlag, 1977.

[LT] E. Lerman and S. Tolman. Hamiltonian torus actions on symplectic orbifolds and toric varieties. Trans. Amer. Math. Soc. 349 (1997) no. 10, 4201-4230.

[MS] E. Miller and B. Sturmfels. Combinatorial Commutative Algebra. Graduate Texts in Mathematics 227, Springer-Verlag, 2005.

[St] R. Stanley. The number of faces of a simplicial convex polytope. Adv. in Math. 35 (1980), no. 3, $236-238$. 